\title{
COMMUTATIVITY OF INTERTWINING OPERATORS
}

\author{
BY A. W. KNAPP ${ }^{1}$ \\ Communicated by Elias Stein, March 29, 1973
}

For each representation of the principal series of a connected semisimple Lie group of matrices, one knows from [1] and [3] a linear basis for the algebra of bounded linear operators that commute with the representation. The relationships among the invariant subspaces for the representation require knowledge of the multiplication in this algebra, however, and the best that was known was the result of [3] that the basic operators are all unitary of order two and commute modulo \pm signs. $^{2}$ Since the full 2-by-2 matrix algebra is generated by four such operators, this information does not completely determine the commuting ring.

Our main result here is that the commuting ring is abelian. This fact, together with the information in [3], enables us to describe the structure of the commuting ring in the theorem below. In particular, the irreducible constituents of any principal series representation are inequivalent.

Let the semisimple group of matrices be $G$, let $G=A N K$ be an Iwasawa decomposition, and let $M$ and $M^{\prime}$ be, respectively, the centralizer and the normalizer of $A$ in $K$. The principal series representation $U(\sigma, \lambda)$ (with the $G$ variable suppressed) is the unitary representation induced from the representation $\sigma \otimes \lambda \otimes 1$ of the closed subgroup $M A N$, where $\sigma$ is an irreducible unitary representation of $M$ and $\lambda$ is a unitary character of $A$.

The group $M^{\prime}$ normalizes $M$, as well as $A$, and therefore acts on both representations $\sigma$ of $M$ and characters $\lambda$ of $A$. If $w$ is in $M^{\prime}$, the character $w \lambda$ and the equivalence class $[w \sigma]$ depend only on the image $[w]$ of $w$ in $W=M^{\prime} / M$, the Weyl group. For each pair $(\sigma, \lambda)$, E. M. Stein and the author [1] constructed a unitary operator $\mathscr{A}(w, \sigma, \lambda)$ satisfying

$$
U(w \sigma, w \lambda) \mathscr{A}(w, \sigma, \lambda)=\mathscr{A}(w, \sigma, \lambda) U(\sigma, \lambda)
$$

and some further properties summarized in [3]. Let

$$
W_{\sigma, \lambda}=\{p \in W \mid[p \sigma]=[\sigma] \text { and } p \lambda=\lambda\} .
$$

If $w$ is in $M^{\prime}$ and $[w]$ is in $W_{\sigma, \lambda}$, then it is possible to extend $\sigma$ to a representation on the same vector space of the group generated by $M$ and $w$; the definition of $\sigma(w)$ is unique except for a scalar factor. Then the operator

AMS (MOS) subject classifications (1970). Primary 22E30, 22E45; Secondary 17B20, 20G20, 22D30, 22E15.

${ }^{1}$ Partly in collaboration with E. M. Stein. Supported by NSF Grant GP 28251.

${ }^{2} \mathrm{~A}$ footnote added in proof to [3] alludes to the present result. 
$\sigma(w) \mathscr{A}(w, \sigma, \lambda)$ commutes with $U(\sigma, \lambda)$ and depends only on $[w]$. We can thus speak of $\sigma(p) \mathscr{A}(p, \sigma, \lambda)$ for $p$ in $W_{\sigma, \lambda}$. The theorem asserts the commutativity of the operators $\sigma(p) \mathscr{A}(p, \sigma, \lambda)$ and then combines this conclusion with the results of [2] and [3]. It is stated in terms of a subgroup $R_{\sigma, \lambda}$ of $W_{\sigma, \lambda}$ that is defined in [2] and in [3], and it uses the unpublished theorem of Harish-Chandra quoted in [3].

THEOREM. The operators $\sigma(r) \mathscr{A}(r, \sigma, \lambda)$ for $r$ in $R_{\sigma, \lambda}$ are a linear basis for the commuting ring of $U(\sigma, \lambda)$. The ambiguous scalar factors in the definitions of the $\sigma(r)$ can be chosen so that the operators $\sigma(r) \mathscr{A}(r, \sigma, \lambda)$ are closed under multiplication and form a group isomorphic to a direct sum $\sum \boldsymbol{Z}_{2}$, with the number of summands bounded by the dimension of $A$.

The exact dimension of the commuting ring (i.e., the order of $R_{\sigma, \lambda}$ ) is given by a formula in [3] in terms of certain factors related to the Plancherel measure of $G$, but it is not apparent from the formula that the dimension is a power of two.

The new part of the theorem, the commutativity of the operators, turns out to be a completely algebraic question. In fact, the operators $\mathscr{A}(w, \sigma, \lambda)$ satisfy a cocycle relation

$$
\mathscr{A}\left(w_{1} w_{2}, \sigma, \lambda\right)=\mathscr{A}\left(w_{1}, w_{2} \sigma, w_{2} \lambda\right) \mathscr{A}\left(w_{2}, \sigma, \lambda\right),
$$

and $R_{\sigma, \lambda}$ is known from [3] to be $\sum \boldsymbol{Z}_{2}$. Putting these facts together, we find that if $[u]$ and $[v]$ are in $W_{\sigma, \lambda}$ and commute, then $\sigma(u) \mathscr{A}(u, \sigma, \lambda)$ and $\sigma(v) \mathscr{A}(v, \sigma, \lambda)$ commute if and only if

$$
\sigma(u) \sigma(v) \sigma(u)^{-1} \sigma(v)^{-1}=\sigma\left(u v u^{-1} v^{-1}\right) .
$$

The validity of $(*)$ depends only on $[u]$ and $[v]$, and it does not depend on the ambiguous scalar factors in the definitions of $\sigma(u)$ and $\sigma(v)$.

We shall indicate some steps in the proof of the theorem. After some preliminary reductions, we may assume that $\lambda=1$ and that the highest weight of $\sigma$ is dominant in the sense of [3]. In this case let $\Delta_{0}$ be the set of restricted roots $\alpha$ for which the root space of $\alpha$ has odd dimension. $\Delta_{0}$ is a closed subsystem of the restricted roots, and we let $S$ be its Weyl group, which we can regard as a subgroup of $W$.

LEMMA $1 . R_{\sigma, 1}$ is contained in $S$.

LeMMa 2. Any two commuting elements of $S$ of order 2 have commuting representatives in $M^{\prime}$ that centralize the identity component of $M$.

Combining the lemmas, we let $u$ and $v$ be the representatives of two elements of $R_{\sigma, 1}$. The right side of $(*)$ is the identity since $u$ and $v$ commute. Since $u$ and $v$ commute with the identity component of $M$ and $\sigma$ is 
irreducible on the identity component, $\sigma(u)$ and $\sigma(v)$ are scalar. Thus the left side of $(*)$ is the identity, and the theorem follows.

Lemma 2 is proved by reducing it to the case that $G$ is split over $\boldsymbol{R}$. There is a canonical way of defining a reductive subgroup $G_{0}$ of $G$ that is split over $R$, has as Cartan subalgebra the Lie algebra of $A$, and has $\Delta_{0}$ as root system. Then $S$ is the Weyl group of $G_{0}$. Lemma 2 follows by applying Lemma 3 below to $G_{0}$.

LEMMA 3. If $G$ is split over $\boldsymbol{R}$, then any two commuting elements of order 2 in the Weyl group have commuting representatives in $M^{\prime}$.

The proof of Lemma 3 is constructive but messy.

\section{REFERENCES}

1. A. W. Knapp and E. M. Stein, Intertwining operators for semisimple groups, Ann. of Math. (2) 93 (1971), 489-578.

2. - Irreducibility theorems for the principal series, Conference on Harmonic Analysis, Lecture Notes in Math., vol. 266, Springer-Verlag, Berlin and New York, 1972, pp. 197-214.

3. A. W. Knapp, Determination of intertwining operators, Summer Institute in Harmonic Analysis 1972, Proc. Sympos. Pure Math., vol. 26, Amer. Math. Soc., Providence, R.I. (to appear).

Department of Mathematics, Cornell University, Ithaca, New York 14850 